\title{
Evaluación de la toxicidad del aceite esencial de Aloysia triphylla Britton (cedrón) y de la actividad anti-Trypanosoma cruzi del citral, in
}

\author{
In vivo evaluation of Aloysia triphylla britton (lemon verbena) essential oil toxicity and citral \\ anti-Trypanosma cruzi activity
}

\author{
Juan Rojas Armas ${ }^{1, a}$, Olga Palacios Agüero, ${ }^{1, b}$, José Manuel Ortiz Sánchez ${ }^{1, c}$, \\ Leavit López de la Peña ${ }^{2, d}$ \\ ${ }^{1}$ Facultad de Medicina, Universidad Nacional Mayor de San Marcos (UNMSM), Lima, Perú. \\ ${ }^{2}$ Instituto Nacional de Salud, Lima, Perú. \\ ${ }^{a}$ Profesor Asociado, Ciencias Dinámicas, Instituto de Investigaciones Clínicas; ${ }^{b}$ Profesor Emérito, Instituto de Medicina Tropical; \\ ${ }^{c}$ Profesor Principal, Ciencias Dinámicas, Instituto de Biología Andina; ${ }^{\circledR}$ Químico Farmacéutico; Estudiante de Posgrado, Facultad
} de Farmacia y Bioquímica, UNMSM.

\begin{abstract}
Resumen
Introducción: Existe escasa investigación en enfermedades olvidadas. Las plantas medicinales son una potencial fuente de compuestos antimicrobianos. Objetivos: Determinar la toxicidad del aceite esencial de Aloysia triphylla y la actividad del citral contra Trypanosoma cruzi en ratones. Diseño: Estudio experimental preclínico in vivo. Institución: Facultad de Medicina, Universidad Nacional Mayor de San Marcos, Lima, Perú. Material: Ratones albinos. Intervenciones: La toxicidad aguda oral a dosis única fue evaluada en ratas albinas. Para la actividad tripanocida se utilizaron ratones asignados a los siguientes grupos: infectados y no tratados (G1), infectados y tratados con citral en dosis de 50, 150 y $300 \mathrm{mg} / \mathrm{kg} / \mathrm{dia}$ (G2, G3 y G4, respectivamente), infectados y tratados con benznidazol $100 \mathrm{mg} / \mathrm{kg}$ (G5) y no infectados y no tratados $(\mathrm{G} 6)$. La parasitemia fue determinada individualmente cada 2 dias por microscopia directa. En los dias 14 , 21 y 28 post infección, cinco ratones de cada grupo fueron sacrificados y los corazones procesados para análisis histopatológico. Principales medidas de resultados: Signos de toxicidad y mortalidad, y parasitemia. Resultados: La dosis límite de $2000 \mathrm{mg} / \mathrm{kg} \mathrm{no}$ provocó signos ni sintomas de toxicidad y los estudios anatomopatológicos macroscópicos y microscópicos no mostraron alteración de los órganos estudiados. La parasitemia fue reducida significativamente con la dosis de $300 \mathrm{mg} / \mathrm{kg}$ en los dias $16^{\circ} 18^{\circ}$ y $20^{\circ}$ post infección $(p<0,05)$. El número de nidos de amastigotes y de infiltrados inflamatorios en corazón fueron reducidos en $67,7 \%$ y $51,7 \%$, respectivamente, con $300 \mathrm{mg} / \mathrm{kg}$ en el día $28^{\circ}$. Conclusiones: El aceite esencial de Aloysia triphylla es calificado como no tóxico y el citral en dosis de $300 \mathrm{mg} / \mathrm{kg}$ tuvo actividad contra Trypanosoma cruzi en ratones.
\end{abstract}

Palabras clave: Trypanosoma cruzi, aceite esencial, cedrón, toxicidad, citral.

\section{Abstract}

Introduction: There is limited research on neglected diseases. Medicinal plants are potential sources of antimicrobial compounds. Objectives: To determine the toxicity of Aloysia triphylla essential oil and citral activity against Trypanosoma cruzi in mice. Design: Experimental study in vivo, preclinical. Setting: Faculty of Medicine, Universidad Nacional Mayor de San Marcos, Lima, Peru. Biological material: Albino mice. Main outcome measures: Signs of toxicity and mortality and parasitemia. Interventions: Acute oral toxicity at single dose was evaluated in albino rats. For trypanocidal activity mice were assigned to the following groups: untreated infected (G1), infected and treated with citral at doses 50,150 and $300 \mathrm{mg} / \mathrm{kg} / \mathrm{day}$ (G2, G3 and G4 respectively), infected and treated with benznidazole $100 \mathrm{mg} / \mathrm{kg}$ (G5), and uninfected and untreated (G6). Parasitemia was determined individually every 2 days by direct microscopy. In days 14, 21 and 28 post infection five mice from each group were sacrificed and their hearts processed for histopathology. Results: The limit dose of $2000 \mathrm{mg} / \mathrm{kg}$ did not cause signs or symptoms of toxicity and macro and microscopic anatomopathology did not show alterations in the organs studied. Parasitemia was significantly reduced at dose of $300 \mathrm{mg} / \mathrm{kg}$ at days 16, 18, and 20 post infection (p $<0.05$ ); the number of amastigote nests and inflammatory infiltrates in heart were reduced on day 28 by $67.7 \%$ and $51.7 \%$ respectively with $300 \mathrm{mg} / \mathrm{kg}$. Conclusions: Aloysia triphylla essential oil is qualified as nontoxic and citral at $300 \mathrm{mg} / \mathrm{kg}$ dose had activity against Trypanosoma cruzi in mice.

Keywords: Trypanosoma cruzi, essential oil, cedron, toxicity, citral.

An Fac med. 2015;76(2):129-34 / dx.doi.org/10.15381/anales.v76i2.11137

\section{INTRODUCCIÓN}

En Latinoamérica existen 18 a 20 millones de personas que sufren la enfermedad de Chagas o tripanosomiasis americana, que en la forma crónica constituye una causa importante de miocardiopatía, megaesófago, megaco- lon y muerte ${ }^{(1)}$. Solamente existen dos fármacos para el tratamiento de la enfermedad de Chagas, benznidazol y nifurtimox, los cuales son útiles solo para la fase aguda; además de ser ineficaces son tóxicos y se requiere administrarlos por largo tiempo ${ }^{(2)}$. Por otra parte, las poblaciones afectadas por esta enferme- dad son socioeconómicamente pobres, lo cual no estimula a la industria farmacéutica a investigar en este campo, dado que no tiene buenas perspectivas de rentabilidad y lucro; por eso, la Organización Mundial de la Salud considera a la enfermedad de Chagas como una de las enfermedades olvidadas ${ }^{(3)}$. 
Por tal motivo, es urgente la búsqueda de nuevos agentes terapéuticos para esta enfermedad y los recursos naturales son una alternativa viable.

Aloysia triphylla Britton es una planta espontánea de América del Sur, originaria del Perú ${ }^{(4)}$; popularmente se la conoce como 'cedrón', 'cidrón', 'limón verbena', 'verbena', 'yerba luisa' o 'hierba de la princesa', según el país o la región ${ }^{(5)}$. Se ha demostrado que el aceite esencial de Aloysia triphylla presenta importante actividad antimicrobiana contra Staphylococcus aureus, Staphylococcus epidermidis, Bacillus cereus, Escherichia coli, Klebsiella pneumoniae, Proteus mirabilis, Enterobacter aerogenes, Klebsiella ozaenae, Enterococcus sp, Bacillus subtilis y Candida albicans ${ }^{(6,7)}$; también produce inhibición in vitro de la replicación de 4 serotipos del virus del dengue ${ }^{(8)}$, y notable actividad antioxidante, mayor que la vitamina $\mathrm{E}^{\left({ }^{(9)}\right.}$. En estudios previos hemos demostrado que el aceite esencial de Aloysia triphylla inhibió el crecimiento in vitro de epimastigotes de T. cruzi con una $\mathrm{CI}_{50}$ de 96,49 $\mu \mathrm{g} / \mathrm{mL}^{(10)}$, así como la actividad antiTrypanosoma cruzi en ratones ${ }^{(11)}$.

El citral es el principal componente del aceite esencial de Aloysia triphylla Britton y de otras plantas aromáticas como Cymbopogon citratus, Melissa officinalis, Verbena officinalis ${ }^{(12)}$. Se ha informado diversas actividades biológicas del citral, tales como antiinflamatoria, en el modelo de edema de pata inducido por carragenina ${ }^{(13,14)}$, supresión de la COX-2 y activación de PPAR $\gamma^{(15)}$, inhibición de la producción de óxido nítrico inducida por lipopolisacárido ${ }^{(16)}$, actividad antinociceptiva ${ }^{(17)}$, vasodilatadora en anillos aórticos de rata ${ }^{(18)}$, espasmolítica ${ }^{(13,19)}$, sedante y relajante motora ${ }^{(20)}$, actividad antiproliferativa en células NB4 de leucemia promielocítica vía la inducción de apoptosis ${ }^{(21)}$, proapoptótica en leucemia mielocítica crónica probablemente por activación directa de procaspasa $3^{(22)}$, inducción de apoptosis en varias líneas celulares de cáncer hematopoyético ${ }^{(12)}$, e inhibición del crecimiento de células MCF-7 de cáncer de mama humano con detención del ciclo celular en la fase $G(2) / M{ }^{(23)}$. También mostró efecto anti-clastogénico al inhibir la formación de micronúcleos inducido por níquel (24). Además, se ha demostrado actividad antimicrobiana como inhibición del crecimiento de hifas de Trichophyton mentagrophytes -en donde se observó que la membrana celular y las organelas fueron dañadas irreversiblemente por el citral (25) , , potente actividad in vitro contra Candida spp. ${ }^{(26)}$, actividad antiviral contra virus de la fiebre amarilla ${ }^{(27)}$ y herpes virus simple tipo 1 (HSV-1) in vitro ${ }^{(28)}$.

Basados en estos importantes antecedentes de las actividades biológicas de Aloysia triphylla Britton y la necesidad de establecer la seguridad como prueba preclínica indispensable, nos propusimos evaluar la toxicidad aguda oral del aceite esencial de Aloysia triphylla Britton y la actividad anti-Trypanosoma cruzi del principal componente de este aceite esencial, el citral, en un modelo murino.

\section{MÉTODOS}

La planta Aloysia triphylla Britton fue colectada en la ciudad de Huaraz, Perú; una muestra fue llevada al Museo de Historia Natural de la UNMSM para su identificación taxonómica (constancia $\mathrm{N}^{\circ}$ 070-USM-2010). El aceite esencial de la planta fue obtenido por arrastre con vapor de agua en un aparato tipo clevenger ${ }^{(29)}$, para lo cual se utilizó las hojas frescas; el aceite esencial fue separado y deshidratado con $\mathrm{Na}_{2} \mathrm{SO}_{4}$ anhidro, se filtró y guardó en un frasco de vidrio color ámbar bajo refrigeración a una temperatura de $4 \stackrel{\circ}{\mathrm{C}}$ hasta su uso.

La toxicidad aguda a dosis única se evaluó mediante el método de OECD (Organisation for Economic Cooperation and Development), directriz $420^{(30)}$. Se utilizó ratas albinas hembras de la raza Holtzman, de $180 \pm 20 \mathrm{~g}$ de peso corporal y 7 semanas de edad, adquiridas en el Instituto Nacional de Salud, las cuales fueron aleatoriamente asignadas a 4 grupos $(n=5)$. Un grupo fue con- trol y solo recibió el vehículo (dimetil sulfóxido (DMSO) (Sigma-Aldrich ${ }^{\circledR}$ ) al 1\% en agua), y los 3 restantes recibieron el aceite esencial de cedrón en dosis única de 2000,300 y $50 \mathrm{mg} / \mathrm{kg}$ de peso corporal, respectivamente. Los animales fueron observados individualmente durante los primeros 30 minutos, con especial atención durante las primeras 4 horas y diariamente hasta los 14 días del experimento, recogiéndose signos y síntomas de toxicidad. Las observaciones estuvieron dirigidas a la determinación de muerte y tiempo de ocurrencia de la misma, signos y síntomas de toxicidad incluyendo su comienzo y duración, además de cambios en la piel, pelaje, membranas de mucosas y ojos, en el sistema respiratorio, circulatorio, nervioso central y autónomo, en la actividad somatomotora y comportamiento. Se prestó especial atención a la potencial ocurrencia de temblor, convulsiones, salivación, diarrea, letargo, somnolencia y coma. Se controló el peso de los animales en los días 0,7 y 14. Al concluir el experimento se sacrificó los animales por inhalación de éter etílico para la posterior necropsia y estudio anatomopatológico macroscópico del estómago, el hígado, el bazo, los pulmones, los riñones, el esófago y el intestino delgado. Finalmente, se realizó el examen microscópico de estos órganos.

Para la determinación de la actividad anti-Trypanosoma cruzi en ratones, como parásitos se usó tripomastigotes sanguíneos cosechados por punción cardíaca de ratones albinos infectados por Trypanosoma cruzi en el pico de parasitemia.

Para la infección in vivo, se utilizó ratones albinos machos, cepa BALB/c, de 25 a $30 \mathrm{~g}$ de peso corporal y 5 semanas de edad; fueron obtenidos del Instituto Nacional de Salud, mantenidos bajo un ciclo de luz/oscuridad de 12/12 horas y se les permitió una semana de aclimatación antes del experimento con alimento y agua ad libitum. La infección se ejecutó por inyección intraperitoneal de $10^{4}$ tripomastigotes sanguíneos. 
Tabla 1. Variación del peso corporal $(\bar{x} \pm \mathrm{EE})$ durante 14 dias, después de la administración de dosis únicas de aceite esencial de Aloysia triphylla.

\begin{tabular}{cccc} 
& \multicolumn{3}{c}{ Día } \\
\cline { 2 - 4 } Vehículo & 0 & 7 & 14 \\
$2000 \mathrm{mg} / \mathrm{kg}$ & $187,8 \pm 1,3$ & $216,0 \pm 4,7$ & $225 \pm 5,5$ \\
$300 \mathrm{mg} / \mathrm{kg}$ & $184,7 \pm 6,0$ & $198,5 \pm 2,7$ & $220 \pm 3,1$ \\
$50 \mathrm{mg} / \mathrm{kg}$ & $185,0 \pm 4,6$ & $208,0 \pm 6,4$ & $218 \pm 6,1$ \\
\hline
\end{tabular}

Los animales fueron asignados aleatoriamente a los siguientes grupos ( $\mathrm{n}$ $=15)$ : infectados y no tratados (G1), infectados y tratados con citral en dosis de 50,150 y $300 \mathrm{mg} / \mathrm{kg} / \mathrm{día}(\mathrm{G} 2, \mathrm{G} 3$ y G4, respectivamente), infectados y tratados con benznidazol $100 \mathrm{mg} / \mathrm{kg}$ (G5) y no infectados y no tratados (G6).

El citral fue adquirido de SigmaAldrich ${ }^{\circledR}$. Fue disuelto en dimetilsulfóxido (DMSO) al 1\% y se administró por vía intragástrica, mediante sonda orogástrica, en dosis de 50, 150 y 300 mg/kg/día a los grupos G2, G3 y G4, respectivamente, desde el 7 al 28 día post infección (dpi). El benznidazol, en suspensión, fue administrado en dosis de $100 \mathrm{mg} / \mathrm{kg} /$ día (G5), de manera similar a los 3 grupos anteriores, mientras que el grupo control (G1) solo recibió el vehículo en igual volumen.
La parasitemia fue observada individualmente cada 2 días por microscopia directa a 400X, contando los parásitos en $5 \mu \mathrm{L}$ de sangre obtenida de la cola.

En los dpi 14, 21 y 28 se sacrificó 5 ratones de cada grupo, se removió los corazones, se los cortó longitudinalmente, se lavó en buffer fosfato salino (PBS) helado y fijó en formaldehido al 10\% en buffer fosfato salino (PBS). Las muestras de tejidos fijados fueron deshidratadas y embebidas en parafina. Tres secciones $(3 \mu \mathrm{m})$ de cada muestra fueron coloreadas con hematoxilinaeosina (HE) y fueron analizadas por microscopia de luz. Se determinó el grado de fibrosis, número de nidos de amastigotes y de infiltrados inflamatorios (más de 10 células mononucleares) en al menos 100 campos en cada lámina. Se obtuvo el número promedio de

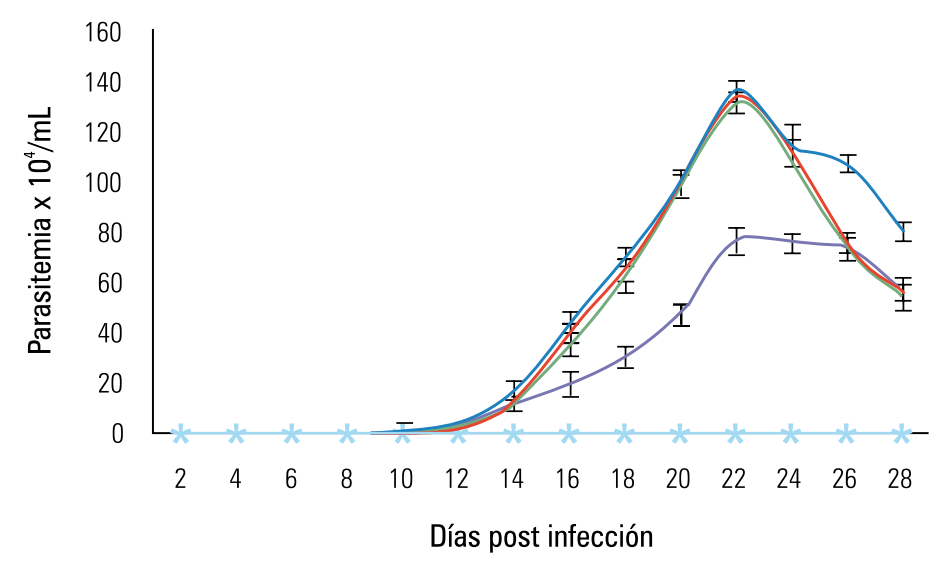

No tratado $\longrightarrow$ Citral $50-$ Citral $150-$ Citral $300 \quad *$ Benznidazol

Figura 1. Efecto del citral sobre la parasitemia por T. cruzi en ratones Balb/c durante 3 semanas de tratamiento a partir del $7^{\circ} \mathrm{dpi}$.

nidos de amastigotes y de infiltrados inflamatorios por campo, con tres secciones de cada corazón.

Los datos obtenidos fueron expresados como media \pm desviación estándar y las comparaciones entre los grupos experimentales se realizaron por ANO. VA de una vía, seguido por una prueba post-hoc de Dunnett. Las medias se consideraron significativamente diferentes cuando $\mathrm{p}<0,05$. Se utilizó el software SPSS versión 19.

\section{RESULTADOS}

La dosis límite de $2000 \mathrm{mg} / \mathrm{kg}$ no provocó signos o síntomas de toxicidad ni muertes en los animales de experimentación. El peso corporal como indicador de toxicidad no varió de manera significativa a lo largo de las dos semanas de evaluación y la tendencia a la ganancia de peso fue normal (tabla 1).

Los estudios anatomopatológicos macroscópicos y microscópicos no mostraron alteración de los órganos estudiados.

El tratamiento con citral en dosis de 50 y $150 \mathrm{mg} / \mathrm{kg}$ no produjo variación significativa sobre la parasitemia a lo largo de todo el periodo de tratamiento; sin embargo, con la dosis de $300 \mathrm{mg} /$ $\mathrm{kg}$ se evidenció una reducción significativa de la parasitemia en los dpi 1618 y $20(\mathrm{p}<0,05)$, mientras que en el 22 dpi, no obstante la disminución desde $136,3 \pm 4,2$ a $76,8 \pm 5,5$ tripomastigotes $\times 10^{4} / \mathrm{mL}$ no fue significativa ( $\mathrm{p}=$ 0,06 , con la prueba de Dunnett), a pesar de representar $43,7 \%$ de reducción (figura 1).

El número de nidos de amastigotes en tejido cardiaco de los ratones disminuyó por efecto del tratamiento con las diversas dosis del citral; aunque no fue significativa, dicha reducción fue mayor con la dosis de $300 \mathrm{mg} / \mathrm{kg}$ que en el $28^{\circ}$ dpi redujo en $67,7 \%$ la carga parasitaria (figuras 2 y 3 ).

El número de infiltrados inflamatorios en tejido cardiaco de los ratones también disminuyó con el tratamiento 


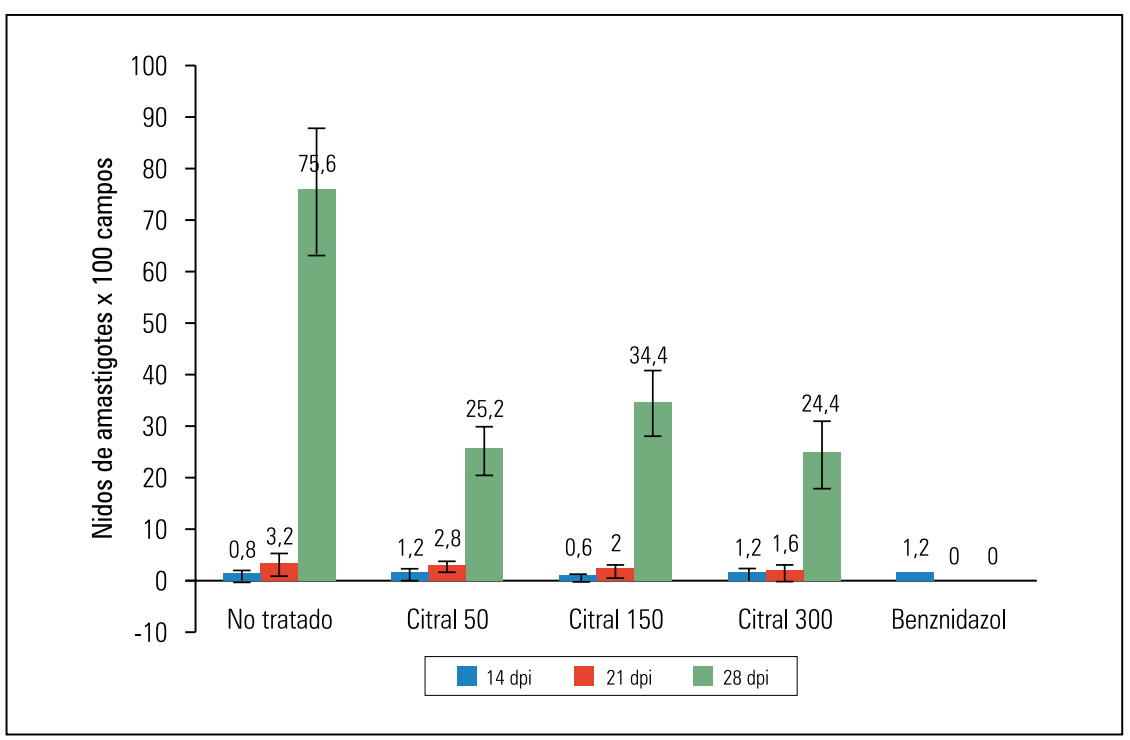

Figura 2. Efecto del citral sobre el número de amastigotes en tejido cardiaco de ratones infectados con 104 tripomastigotes de T. cruzi.

con citral, siendo mayor con la dosis de $300 \mathrm{mg} / \mathrm{kg}$ que en el $28^{\circ}$ dpi redujo de $106 \pm 13,2$ a $51,2 \pm 14,3$ infiltrados inflamatorios x 100 campos ( $\mathrm{p}<$ $0,05)$, representando una reducción del $51,7 \%$ (figura 4 ).

\section{DISCUSIÓN}

La existencia de plantas con un elevado potencial terapéutico constituye una alternativa farmacológica de marcado interés en el tratamiento de muchas enfermedades. De ahí la importancia de realizar estudios preclínicos con el

propósito de detectar posibles efectos tóxicos post administración. Dentro de la batería de ensayos de primera barrera se encuentran los estudios de toxicidad a dosis única, imprescindibles en la estimación del potencial tóxico de una sustancia, referido como el estudio cualicuantitativo de los fenómenos tóxicos y de su aparición en función del tiempo tras la administración de una dosis única de la sustancia o de varias dosis fraccionadas en el transcurso de 24 horas. En este sentido, el procedimiento de dosis fijas fue adoptado en la Guía No. 420 de la OECD como alternativa a la prueba clásica de toxicidad aguda. ${ }^{(30)}$.

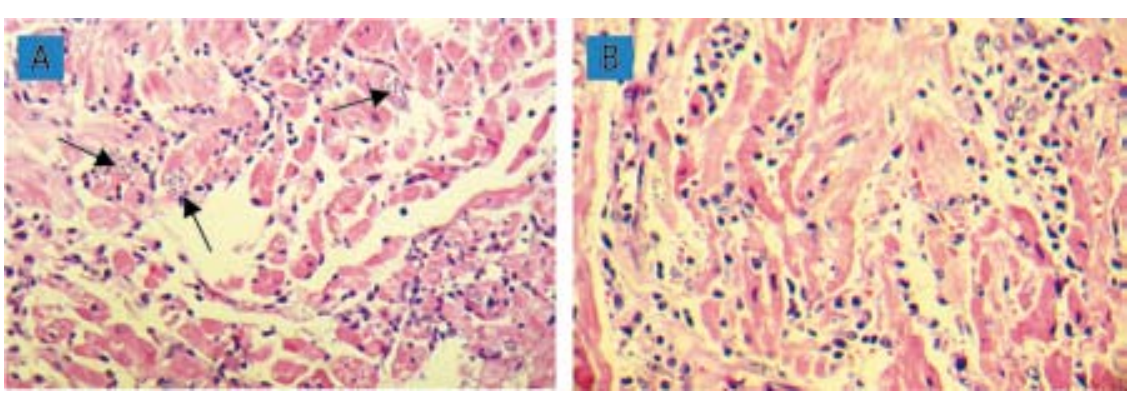

Figura 3. Tejido cardiaco de ratones BALB/c infectados con Trypanosoma cruzi, en el 28 dpi, después del tratamiento con citral a partir del 7 dpi. (Coloración HE 400X). [A] Nidos de amastigotes (flechas) e infiltrados inflamatorios en el grupo infectado con T. cruzi y sin tratamiento (G1). [B] Grupo infectado con T. cruzi y tratado con citral $300 \mathrm{mg} / \mathrm{kg} / \mathrm{dia}$.
El ensayo de toxicidad oral aguda proporciona información sobre los efectos adversos que pueden acaecer, en un breve período de tiempo, como consecuencia de la ingestión de una única dosis de la sustancia que se estudia. Desde el punto de vista regulatorio se acepta que no es necesario definir un valor puntual de la dosis letal media $\left(\mathrm{DL}_{50}\right)$ para una sustancia y que resulta prácticamente suficiente ubicar dicha dosis en un rango ${ }^{(31)}$. Las regulaciones para la ejecución de este tipo de estudio de toxicidad aguda plantean que si no existen informes anteriores de toxicidad de la(s) sustancia(s) que se investiga(n) es posible utilizar una dosis límite de $2000 \mathrm{mg} / \mathrm{kg}$ de masa corporal ${ }^{(32)}$, lo que se ajusta a nuestro estudio, dado que para el aceite esencial de Aloysia triphylla no se ha señalado toxicidad en su extenso uso tradicional.

En nuestro estudio, no se observaron signos ni síntomas de toxicidad y tampoco se produjo la muerte de ningún animal de experimentación, así como tampoco se observaron alteraciones macroscópicas o microscópicas de los órganos con la dosis máxima de 2000 $\mathrm{mg} / \mathrm{kg}$. Además, el peso corporal de las ratas no varió de manera significativa a lo largo del experimento y la tendencia a la ganancia de peso fue normal (tabla 1); esto nos permite afirmar que la $\mathrm{DL}_{50}$ del aceite esencial de Aloysia triphylla se encuentra por encima de $2000 \mathrm{mg} / \mathrm{kg}$, por lo que es calificado, según el Sistema Global Armonizado, como 'No clasificado' ('No tóxico').

La parasitemia alcanzó el pico en el $22^{\circ}$ día post infección, lo cual coincide con los hallazgos previos para la cepa peruana de Trypanosoma cruzi ${ }^{(11)}$. En ese mismo estudio demostramos que la parasitemia en el $22^{\circ}$ dpi se redujo $85,4 \%$ con el tratamiento con aceite esencial de Aloysia triphylla en dosis de $250 \mathrm{mg} / \mathrm{kg}$. Sin embargo, en la presente investigación, el citral en dosis de $300 \mathrm{mg} / \mathrm{kg}$ redujo $43,7 \%$ de parasitemia (figura 1), lo que indica que el citral, principal componente del aceite esencial de Aloysia triphylla, no tiene la misma eficacia a pesar de haberse utili- 


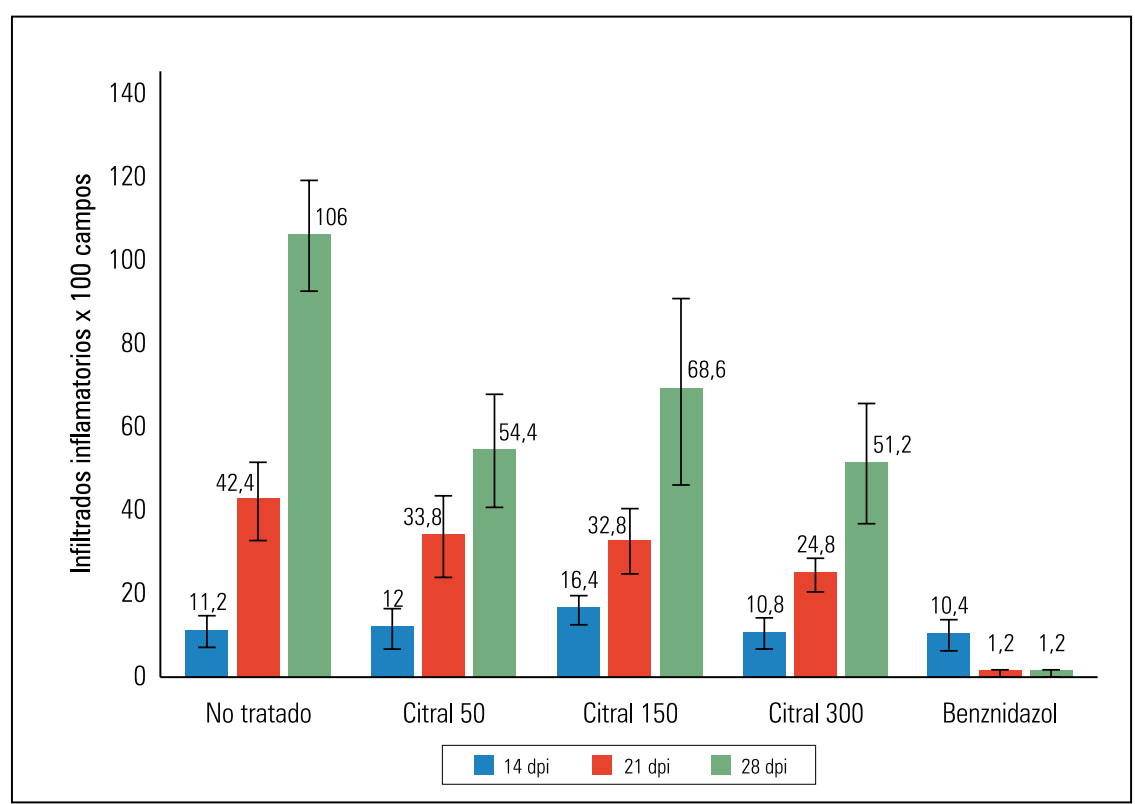

Figura 4. Efecto del citral sobre el número de infiltrados inflamatorios en tejido cardiaco de ratones infectados con 104 tripomastigotes de $T$. cruzi.

zado una dosis mayor, lo cual evidencia que el efecto anti-Trypanosoma cruzi se debería además a algunos de los otros componentes del aceite que hacen sinergismo con el citral.

La actividad del citral contra Trypanosoma cruzi en ratones, evidenciado por la disminución de las formas tripomastigotes en sangre, se corresponde con los hallazgos de una disminución de las formas amastigotes (figuras 2 y 3) y focos inflamatorios (figura 4) en tejido cardiaco de los ratones al final del experimento. Estas observaciones guardan relación con la investigación de Cardoso y Soares (2010) quienes demostraron in vitro que el citral en concentración mayor de $60 \mu \mathrm{g} / \mathrm{mL}$ produce $100 \%$ de lisis de formas epimastigotes y tripomastigotes de Trypanosoma

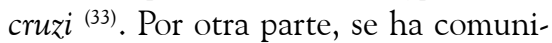
cado que el citral exhibió actividad antiparasitaria contra tripanosomátidos del género Leishmania al inhibir la proliferación de promastigotes y amastigotes axénicos e intracelulares de Leishmania amazonensis ${ }^{(34)}$ y promastigotes de Leishmania infantum, Leishmania tropica y Leishmania major, con considerables alteraciones ultraestructurales, princi-
3. Hotez P, Molyneux D, Fenwick A, Kumaresan J, Ehrlich S, Sachs J, et al. Control of neglected tropical diseases. N Engl J Med. 2007;357:1018-27.

4. Diaz O, Durán D, Martinez J, Stashenko E. Estudio comparativo de la composición química de los aceites esenciales de Aloysia triphylla L' HER BRITTON cultivada en diferentes regiones de CoIombia. Scientia et Technica. 2007;33:351-3.

5. Gattuso S, Baren C, Gil A, Bandoni A, Ferraro G, Gattuso M. Morpho-histological and quantitative parameters in the characterization of lemon verbena (Aloysia citriodora palau) from Argentina. Boletin Latinoamericano y del Caribe de Plantas Medicinales y Aromáticas. 2008;7(4):190-8.

6. Oliva M, Beltramino E, Gallucci M, Casero C, Zygadlo J, Demo M. Antimicrobial activity of essential oils of Aloysia triphylla (L`Her.) Britton from different regions of Argentina. Boletín Latinoamericano y del Caribe de Plantas Medicinales y Aromáticas. 2010;9(1):29-37.

7. Rojas L, Velasco J, Díaz T, Gil R, Carmona J, Usubillaga A. Composición quimica y efecto antibacteriano del aceite esencial de Aloysia triphylla (L'Hér.) Britton contra patógenos genito-urinarios. Boletín Latinoamericano y del Caribe de Plantas Medicinales y Aromáticas. 2010;9(1):56-62.

8. Ocazionez R, Meneses R, Torres F, Stashenko E. Virucidal activity of Colombian Lippia essential oils on dengue virus replication in vitro. Memorias do Instituto Oswaldo Cruz. 2010;105(3):304-9.

9. Stashenko E, Jaramillo B, Martínez J. Comparación de la composición quimica y de la actividad antioxidante in vitro de los metabolitos secundarios volátiles de plantas de la familia Verbenaceae. Rev Acad Colomb Cienc. 2003;27(105):579-97.

10. Rojas J, Solís H, Palacios O. Actividad antiTrypanosoma cruzi in vitro de aceites esenciales de diez plantas medicinales. An Fac med. 2010;71(3):161-5.

11. Rojas J, Palacios O, Ronceros S. Efecto del aceite esencial de Aloysia triphylla Britton (cedrón) sobre el Trypanosoma cruzi en ratones. Rev Peru Med Exp Salud Publica. 2012;29(1):61-8.

12. Dudai N, Weinstein $Y$, Krup M, Rabinski T, Ofir R. Citral is a new inducer of caspase-3 in tumor cell lines. Planta Med. 2005;71(5):484-8.

13. Ponce $H$, Fernández $E$, Ortiz $M$, Ramírez $M$, Cruz D, Pérez N, et al. Spasmolytic and antiinflammatory effects of Aloysia triphylla and citral, in vitro and in vivo studies. J. Smooth Muscle Res. 2010;46(6):309-19.

14. Ortiz MI, González-Garcia MP, Ponce-Monter HA, Castañeda-Hernández G, Aguilar-Robles P. Synergistic effect of the interaction between naproxen and citral on inflammation in rats. Phytomedicine. 2010;18(1):74-9.

15. Katsukawa M, Nakata R, Takizawa Y, Hori K, Takahashi S, Inoue H. Citral, a component of lemongrass oil, activates PPAR $\alpha$ and $\gamma$ and suppresses COX-2 expression. Biochim Biophys Acta. 2010;1801(11):1214-20.

16. Lee HJ, Jeong HS, Kim DJ, Noh YH, Yuk DY, Hong JT. Inhibitory effect of citral on NO production by suppression of iNOS expression and NF-kappa $B$ activation in RAW264.7 cells. Arch Pharm Res. 2008;31(3):342-9.

17. Ortiz MI, Ramirez-Montiel ML, González-Garcia MP, Ponce-Monter HA, Castañeda-Hernández G, Cariño-Cortés R. The combination of naproxen and citral reduces nociception and gastric damage in rats. Arch Pharm Res. 2010;33(10):1691-7.

18. Lopes S, Mesquita A, Takashi R, Coelho M, Zapata G. Vasodilator activity of the essential oil 
from aerial parts of Pectis brevipedunculata and its main constituent citral in rat aorta. Molecules. 2013;18:3072-85.

19. Chitra R, Mui S, Ismail R. Spasmolytic effect of citral and extracts of Cymbopogon citratus on isolated rabbit ileum. J. Smooth Muscle Res. 2011;47(5):143-6.

20. do Vale TG, Furtado EC, Santos JG Jr, Viana GS Central effects of citral, myrcene and limonene, constituents of essential oil chemotypes from Lippia alba (Mill.) n.e. Brown. Phytomedicine. 2002;9(8):709-14.

21. Xia H, Liang W, Song Q, Chen X, Chen X, Hong J. The in vitro study of apoptosis in NB4 cell induced by citral. Cytotechnology. 2013;65(1):49-57.

22. De Martino L, D'Arena G, Minervini MM, Deaglio S, Fusco BM, Cascavilla N, De Feo V. Verbena officinalis essential oil and its component citral as apoptotic-inducing agent in chronic lymphocytic leukemia. Int J Immunopathol Pharmacol. 2009;22(4):1097-104.

23. Chaouki W, Leger DY, Liagre B, Beneytout JL, Hmamouchi M. Citral inhibits cell proliferation and induces apoptosis and cell cycle arrest in MCF-7 cells. Fundam Clin Pharmacol. 2009;23(5):54956.

24. Rabbani SI, Devi K, Khanam S, Zahra N. Citral, component of lemongrass oil inhibits the clastogenic effect of nickel chloride in mouse micronucleus test system. Pak J Pharm Sci. 2006;19(2):108-13.

25. Park MJ, Gwak KS, Yang I, Kim KW, Jeung EB Chang JW, Choi IG. Effect of citral, eugenol, nerolidol and alpha-terpineol on the ultrastructural changes of Trichophyton mentagrophytes. Fitoterapia. 2009;80(5):290-6.
26. de Bona da Silva C, Guterres S, Weisheimer V, Schapoval E. Antifungal activity of the lemongrass oil and citral against Candida spp. Brazilian J Infect Dis. 2008;12(1):63-6.

27. Gómez LA, Stashenko E, Ocazionez RE. Comparative study on in vitro activities of citral, limonene and essential oils from Lippia citriodora and L. alba on yellow fever virus. Nat Prod Commun. 2013;8(2):249-52.

28. Astani A, Reichling J, Schnitzler P. Comparative study on the antiviral activity of selected monoterpenes derived from essential oils. Phytother Res. 2010;24(5):673-9.

29. Lahlou M. Methods to study the phytochemistry and bioactivity of essential oils. J Phytother Res. 2004; 18:435-48.

30. OECD. Organization for Economic Co-operation and Development. Guideline for Testing of Chemicals, Guideline 420.Acute Oral Toxicity-Fixed Dose Procedure. 2001. URL disponible en: http:// www.oecd.org [fecha de acceso 20 de noviembre de 2011].

31. Kennedy GL, Ferenz RL, Burgess BA . Estimation of acute oral toxicity in rats by determination of the approximate lethal dose rather than the LD50. J Appl Toxicol, 1986;6:145-8.

32. Bermudez D, Monteagudo E, Boffill M, Diaz L, Roca A, Betancourt E, et al. Evaluación de la toxicidad aguda de extractos de plantas medicinales por un método alternativo. REDVET. 2007;8(3). [Acceso 19 nov 2012], disponible en http://www.veterinaria. org/revistas/recvet/n030307/030706.pdf

33. Cardoso J, Soares MJ. In vitro effects of citral on Trypanosoma cruzi metacyclogenesis. Mem Inst Oswaldo Cruz. 2010;105(8):1026-32.
34. Santin MR, dos Santos AO, Nakamura CV, Dias Filho BP, Ferreira IC, Ueda-Nakamura T. In vitro activity of the essential oil of Cymbopogon citratus and its major component (citral) on Leishmania amazonensis. Parasitol Res. 2009;105(6):148996

35. Machado M, Pires $P$, Dinis AM, Santos-Rosa $M$, Alves V, Salgueiro $L$, et al. Monoterpenic aldehydes as potential anti-Leishmania agents: activity of Cymbopogon citratus and citral on $\mathrm{L}$. infantum, L. tropica and L. major. Exp Parasitol. 2012;130(3):223-31

Artículo recibido el 29 de setiembre de 2014 y aceptado para publicación el 10 de noviembre de 2014.

Los autores declaran no tener ningún conflicto de interés para la publicación de este artículo.

Correspondencia:

\section{Dr. Juan Rojas Armas}

Instituto de Investigaciones Clínicas, Hospital Nacional Dos de Mayo, Av. Grau cuadra 13, Parque Historia de la Medicina, Lima 1, Perú

Correo electrónico: jprojasarmas@yahoo.com 